\title{
Flops Connect Minimal Models
}

\author{
By
}

\author{
Yujiro KaWAMATA*
}

\begin{abstract}
A result by Birkar-Cascini-Hacon-McKernan together with the boundedness of length of extremal rays implies that different minimal models can be connected by a sequence of flops.

A flop of a pair $(X, B)$ is a flip of a pair $\left(X, B^{\prime}\right)$ which is crepant for $K_{X}+B$ where $B^{\prime}$ is a suitably chosen different boundary. We prove the following:

Theorem 1. Let $f:(X, B) \rightarrow S$ and $f^{\prime}:\left(X^{\prime}, B^{\prime}\right) \rightarrow S$ be projective morphisms from $\mathbf{Q}$-factorial terminal pairs of varieties and $\mathbf{Q}$-divisors such that $K_{X}+B$ and $K_{X^{\prime}}+B^{\prime}$ are relatively nef over $S$. Assume that there exists a birational map $\alpha: X \rightarrow X^{\prime}$ such that $\alpha_{*} B=B^{\prime}$, where the lower asterisk denotes the strict transform. Then $\alpha$ is decomposed into a sequence of flops.

More precisely, there exist an effective Q-divisor $D$ on $X$ such that $(X, B+$ $D)$ is klt and a factorization of the birational map $\alpha$

$$
X=X_{0} \rightarrow X_{1} \rightarrow \cdots \cdots-\rightarrow X_{t}=X^{\prime}
$$

which satisfy the following conditions:

(1) $\alpha_{i}: X_{i-1} \rightarrow X_{i}(1 \leq i \leq t)$ is a flip for the pair $\left(X_{i}, B_{i}+D_{i}\right)$ over $S$, where $B_{i}$ and $D_{i}$ are strict transforms of $B$ and $D$, respectively.

(2) $\alpha_{i}$ is crepant for $K_{X_{i-1}}+B_{i-1}$ in the sense that the pull-backs of $K_{X_{i-1}}+B_{i-1}$ and $K_{X_{i}}+B_{i}$ coincide on a common log resolution.

\footnotetext{
Communicated by S. Mori. Received March 29, 2007. Revised September 1, 2007.

2000 Mathematics Subject Classification(s): 14E30, 14E05, 14J32.

* Department of Mathematical Sciences, University of Tokyo, Komaba, Meguro, Tokyo, 153-8914, Japan.

e-mail: kawamata@ms.u-tokyo.ac.jp
}

(c) 2008 Research Institute for Mathematical Sciences, Kyoto University. All rights reserved.
\end{abstract}


We remark that the boundary $B$ need not be assumed to be big as in [1, Corollary 1.1.3]. For example, a birational map between Calabi-Yau manifolds can be decomposed into a sequence of flops. The number of marked minimal models which are birationally equivalent to a fixed pair is finite if $B$ is big ([1, Corollary 1.1.5]), but it is not the case in general (cf. [4]), where a marked minimal model is a pair consisting of a minimal model and a fixed birational map to it. If we relax the condition for the pairs to being klt, then we should allow crepant blowings up besides flops.

The theorem was already proved in the case $\operatorname{dim} X=3$ and $B=0$; first in [2] assuming the abundance which was proved afterwards, and later in [5] without assumption.

Proof. It is well-known that $\alpha$ is an isomorphism in codimension 1 because $(X, B)$ and $\left(X^{\prime}, B^{\prime}\right)$ are terminal and $K_{X}+B$ and $K_{X^{\prime}}+B^{\prime}$ are relatively nef (cf. [2]). We recall the proof for reader's convenience. Let $\mu: V \rightarrow X$ and $\mu^{\prime}: V \rightarrow X^{\prime}$ be common log resolutions. We write

$$
K_{V}=\mu^{*}\left(K_{X}+B\right)-\mu_{*}^{-1} B+E=\left(\mu^{\prime}\right)^{*}\left(K_{X^{\prime}}+B^{\prime}\right)-\left(\mu_{*}^{\prime}\right)^{-1} B^{\prime}+E^{\prime}
$$

where $E$ and $E^{\prime}$ are effective divisors whose supports coincide with the exceptional loci of $\mu$ and $\mu^{\prime}$, respectively, because $(X, B)$ and $\left(X^{\prime}, B^{\prime}\right)$ are terminal. Assume that there is a prime divisor on $V$ which is contracted by $\mu$ but not by $\mu^{\prime}$. Then it is an irreducible component of $E$ but not of $E^{\prime}$. We set $F=\min \left\{E, E^{\prime}\right\}, \bar{E}=E-F$ and $\bar{E}^{\prime}=E^{\prime}-F$. By the Hodge index theorem, there exists a curve $C$ on $V$ which is contracted by $\mu$ and is contained in $\operatorname{Supp}(\bar{E})$ but not in $\operatorname{Supp}\left(\mu_{*}^{-1} B+\bar{E}^{\prime}\right)$ and such that $(\bar{E} \cdot C)<0$. We have $\mu_{*}^{-1} B \geq\left(\mu_{*}^{\prime}\right)^{-1} B^{\prime}$ because some of the irreducible components of $B$ may be contracted by $\alpha$. Hence

$$
\left.\left(\left(\mu^{\prime}\right)^{*}\left(K_{X^{\prime}}+B^{\prime}\right)+\mu_{*}^{-1} B-\left(\mu_{*}^{\prime}\right)^{-1} B^{\prime}+\bar{E}^{\prime}\right) \cdot C\right) \geq 0 .
$$

But this is a contradiction to

$$
\left(\left(\mu^{*}\left(K_{X}+B\right)+\bar{E}\right) \cdot C\right)<0 .
$$

The case where there is a prime divisor on $V$ which is contracted by $\mu^{\prime}$ but not by $\mu$ is treated similarly as follows. We have $\bar{E}^{\prime} \neq 0$. By the Hodge index theorem applied to $\bar{E}^{\prime}+\mu_{*}^{-1} B-\left(\mu_{*}^{\prime}\right)^{-1} B^{\prime}$, there exists a curve $C$ on $V$ which is contracted by $\mu^{\prime}$ and such that $\left(\left(\bar{E}^{\prime}+\mu_{*}^{-1} B-\left(\mu_{*}^{\prime}\right)^{-1} B^{\prime}\right) \cdot C\right)<0$ but not contained in $\operatorname{Supp}(\bar{E})$. Hence

$$
\left.\left(\left(\mu^{\prime}\right)^{*}\left(K_{X^{\prime}}+B^{\prime}\right)+\bar{E}^{\prime}+\mu_{*}^{-1} B-\left(\mu_{*}^{\prime}\right)^{-1} B^{\prime}\right) \cdot C\right)<0 .
$$


But this is a contradiction to

$$
\left(\left(\mu^{*}\left(K_{X}+B\right)+\bar{E}\right) \cdot C\right) \geq 0
$$

Let $L^{\prime}$ be an effective $f^{\prime}$-ample divisor on $X^{\prime}$, and $L$ its strict transform on $X$. There exists a small positive rational number $l$ such that $(X, B+l L)$ is klt. If $K_{X}+B+l L$ is $f$-nef over $S$, then the base point free theorem implies that there exists a morphism from $X$ over $S$ associated to the $\mathbf{Q}$-divisor $K_{X}+B+l L$, which is equal to $\alpha$ because the direct image sheaf on $S$ of a positive multiple of $K_{X}+B+l L$ is equal to that of an $f^{\prime}$-ample $\mathbf{Q}$-divisor $K_{X^{\prime}}+B^{\prime}+l L^{\prime}$. Hence $\alpha$ is an isomorphism since $X$ is $\mathbf{Q}$-factorial. Therefore we may assume that $K_{X}+B+l^{\prime} L$ is not $f$-nef over $S$ for any $0<l^{\prime} \leq l$.

Let $H$ be an effective divisor on $X$ such that $(X, B+l L+t H)$ is klt and $K_{X}+B+l L+t H$ is $f$-nef for some positive number $t$. We shall run the MMP for the pair $\left(X, B+l^{\prime} L\right)$ over $S$ with scaling of $H$ for some $l^{\prime}$. Since $\alpha$ is an isomorphism in codimension 1, there are only flips in this MMP. The following lemma shows that we can choose extremal rays such that the flips are crepant with respect to $K_{X}+B$.

Let $k$ be a positive integer such that $k\left(K_{X}+B\right)$ is a Cartier divisor. We set $e=\frac{1}{2 k \operatorname{dim} X+1}$.

Lemma 2. (1) There exists an extremal ray $R$ for $(X, B+l L)$ over $S$ such that $\left(\left(K_{X}+B\right) \cdot R\right)=0$.

(2) Let

$$
\begin{gathered}
t_{0}=\min \left\{t \in \mathbf{R} \mid\left(\left(K_{X}+B+l L+t H\right) \cdot R\right) \geq 0 \text { for all extremal rays } R\right. \\
\text { for } \left.(X, B+l L) \text { over } S \text { s.t. }\left(\left(K_{X}+B\right) \cdot R\right)=0\right\} .
\end{gathered}
$$

Then $K_{X}+B+$ elL + et $t_{0} H$ is $f$-nef, and there exists an extremal ray $R$ for $(X, B+$ elL $)$ over $S$ such that $\left(\left(K_{X}+B+\right.\right.$ elL + et $\left.\left.t_{0} H\right) \cdot R\right)=\left(\left(K_{X}+B\right) \cdot R\right)=0$.

Proof. (1) Since $K_{X}+B+$ el $L$ is not nef, there exists an extremal ray $R$ for $(X, B+e l L)$ over $S$. Then $R$ is also an extremal ray for $(X, B+l L)$ because $(X, B)$ is $f$-nef. Since the pair $(X, B+l L)$ is klt, $R$ is generated by a rational curve $C$, which is mapped to a point on $S$, such that

$$
0>\left(\left(K_{X}+B+l L\right) \cdot C\right) \geq-2 \operatorname{dim} X
$$

by [3]. 
We claim that $\left(\left(K_{X}+B\right) \cdot C\right)=0$. Indeed we have otherwise $\left(\left(K_{X}+B\right)\right.$. $C) \geq 1 / k$, hence

$$
\begin{aligned}
& \left(\left(K_{X}+B+e l L\right) \cdot C\right) \\
& =\frac{1}{2 k \operatorname{dim} X+1}\left(\left(K_{X}+B+l L\right) \cdot C\right)+\frac{2 k \operatorname{dim} X}{2 k \operatorname{dim} X+1}\left(\left(K_{X}+B\right) \cdot C\right) \\
& \geq \frac{1}{2 k \operatorname{dim} X+1}(-2 \operatorname{dim} X+2 \operatorname{dim} X)=0
\end{aligned}
$$

a contradiction.

(2) If $K_{X}+B+e l L+e t_{0} H$ is not $f$-nef, then there exists an extremal ray $R$ for $\left(X, B+e l L+e t_{0} H\right)$ over $S$. Then $R$ is also an extremal ray for $\left(X, B+l L+t_{0} H\right)$ because $(X, B)$ is $f$-nef. Since the pair $\left(X, B+l L+t_{0} H\right)$ is klt, $R$ is generated by a rational curve $C$ such that $\left(\left(K_{X}+B+l L+t_{0} H\right) \cdot C\right) \geq$ $-2 \operatorname{dim} X$ by [3]. Then we have

$$
\begin{aligned}
& \left(\left(K_{X}+B+e l L+e t_{0} H\right) \cdot C\right) \\
& =\frac{1}{2 k \operatorname{dim} X+1}\left(\left(K_{X}+B+l L+t_{0} H\right) \cdot C\right)+\frac{2 k \operatorname{dim} X}{2 k \operatorname{dim} X+1}\left(\left(K_{X}+B\right) \cdot C\right) \\
& \geq \frac{1}{2 k \operatorname{dim} X+1}(-2 \operatorname{dim} X+2 \operatorname{dim} X)=0
\end{aligned}
$$

a contradiction. Therefore $K_{X}+B+e l L+e t_{0} H$ is $f$-nef.

Since $B+l L$ is $f$-big, the number of extremal rays for $(X, B+l L)$ over $S$ is finite. Hence there exists such an $R$ that $\left(\left(K_{X}+B+l L+t_{0} H\right) \cdot R\right)=$ $\left(\left(K_{X}+B\right) \cdot R\right)=0$.

We note that the finiteness theorem of extremal rays implies an alternative proof of (1) but not (2). The point is that the number $e$ stays independent of $t_{0}$ during the MMP.

We run the MMP for $(X, B+e l L)$ with scaling of $H$. We take an extremal ray $R$ such that $\left(\left(K_{X}+B+e l L+e t_{0} H\right) \cdot R\right)=\left(\left(K_{X}+B\right) \cdot R\right)=0$. The flip exists by $\left[1\right.$, Corollary 1.4.1]. Since $\left(\left(l L+t_{0} H\right) \cdot R\right)=0$, the pair $\left(X, B+l L+t_{0} H\right)$ remains to be klt after the flip. We also note that $k\left(K_{X}+B\right)$ remains to be a Cartier divisor after the flip by the base point free theorem. Therefore we can continue the process. By the termination theorem of directed flips ([1, Corollary 1.4.2]), we complete our proof. 


\section{References}

[1] C. Birkar, P. Cascini, C. D. Hacon, J. McKernan, Existence of minimal models for varieties of log general type, math.AG/0610203.

[2] Y. Kawamata, Crepant blowing-up of 3-dimensional canonical singularities and its application to degenerations of surfaces, Ann. of Math. (2) 127 (1988), no. 1, 93-163.

[3] $\ldots$, On the length of an extremal rational curve, Invent. Math. 105 (1991), no. 3, 609-611.

[4] , On the cone of divisors of Calabi-Yau fiber spaces, Internat. J. Math. 8 (1997), no. 5, 665-687.

[5] J. Kollár, Flops, Nagoya Math. J. 113 (1989), 15-36. 